\title{
SIZE EFFECTS ON THERMAL PROPERTIES OF THIN METAL FILMS WITH ROUGH SURFACES
}

\author{
C.R. TELLIER \\ Laboratoire de Chronométrie, Electronique et Piézoélectricité, Ecole Nationale Superieure de Mecanique et \\ des Microtechniques, Route de Gray, La Bouloie, 25030 Besancon Cedex, France \\ (Received July 4, 1988; in final form September 12, 1989)
}

By calculating the effects of electronic scattering at rough surfaces by means of a combined Soffer-Cottey model, general theoretical expressions for the size effects in thermal properties of thin metal films are proposed. Simple analytical expressions for the thermoeiectric power, $S_{f}$, and the thermal conductivity, $\mathscr{C}_{\mathrm{f}}$, are given under the assumption that the energy dependence of the electronic relaxation time in bulk material may be written in the form $\tau_{\mathrm{o}} \sim \mathrm{W}^{\mathrm{m}}$. The size effects in the thermoelectric power are found to depend on the value of $\mathrm{m}$. However, a decrease in the overall size effects is observed in all transport parameters with respect to the predictions of classical theories based on the Fuchs-Sondheimer or the Cottey models. A comparison with data on the thermoelectric power of thin copper, silver and tin films from previous experiments by various workers shows that a combined Soffer-Cottey model is convenient to analyze size effects in thermoelectric properties. The values of the r.m.s. surface roughness obtained from data are physically consistent.

\section{INTRODUCTION}

In the last decade a lot of measurements on the thermal galvanomagnetic and electrical properties were performed ${ }^{1}$ on structurally continuous thin metal films. Most of these experimental works were, up till now, interpreted in terms of the earliest theoretical work on the influence of the film thickness on the transport properties which was done by Fuchs $^{2}$ and Sondheimer ${ }^{3}$ (F.S. model). But the F.S. model is based upon the assumption of a constant specularity parameter, $\mathrm{p}$, which is independent of the electron energy, $\mathrm{W}$, and of the angle, $\theta$, of incidence. Hence some authors ${ }^{4-6}$ have presented some more sophisticated models in order to solve the problem of surface scattering when the above assumptions are not fulfilled. In particular the Soffer model ${ }^{5}$ which deals with a theoretical study of the surface roughness effect turns, in the limit where the correlation distance along the surface decreases to zero, to a simple formulation of the specularity parameter $\mathrm{p}$

$p=\exp \left\{-\left(\frac{4 \pi r}{\lambda_{c}}\right)^{2} \cos ^{2} \theta\right\}$

where $r$ is the surface roughness (i.e., the r.m.s. height deviation) and $\lambda_{c}$ is the De Broglie wavelength.

Owing to this simple formulation some attempts have been made in the past few years to derive new equations for the electrical conductivity ${ }^{7,8}$ and for the galvanomagnetic coefficients $^{9,10}$ of thin metal films replacing the constant specularity parameter $\mathrm{p}$ by Eq. 
(1) in the theoretical analysis of the size effect. These theoretical works are based on the use of either the classical F.S. model ${ }^{7,9}$ which involves the use of a computer for a numerical evaluation of the size effect in the transport properties or the Cottey model ${ }^{11}$ which generally offers the possibility to derive analytical expressions ${ }^{8,12}$ for the transport coefficients. But there is up to now no theoretical study devoted to the study of the influence of the surface roughness on the size effect in the thermal properties of metal films.

In view of this lack of theoretical formulations we present in this paper an analysis of the thermal properties of thin metal films starting from the Soffer and the Cottey models. A general treatment is proposed in which analytical expressions for the thermoelectric power, $S_{\mathrm{f}}$, and the thermal conductivity, $\mathscr{C}_{\mathrm{f}}$, are established. Moreover the validity of the Wiedemann and Franz law is discussed in the region of high enough temperatures where we can ignore the phonon thermal conductivity. ${ }^{1}$

\section{GENERAL TREATMENT}

For calculating thermal transport parameters, $S_{\mathrm{f}}$ and $\mathscr{b}_{\mathrm{f}}$, we are concerned with the general expressions for the electrical current density $J_{x}$ and the heat flux density, $U_{x}$, in a thin metal film subjected to an electrical field $\vec{E}\left(E_{x}, 0,0\right)$ and a thermal gradient $\partial T / \partial \vec{r}$ $(\partial \mathrm{T} / \partial \mathrm{x}, 0,0)$ in the $\mathrm{x}$ direction $^{14}$ :

$J_{x}=-\frac{e}{4 \pi^{3}} \iiint v_{x} f^{1} d_{k} d k_{y} d k_{z}$

and

$u_{\mathrm{x}}=\frac{1}{4 \pi^{3}} \iiint \mathrm{v}_{\mathrm{x}}\left(\mathrm{W}-\mathrm{W}_{\mathrm{F}}\right) \mathrm{f}^{1} \mathrm{dk}_{\mathrm{x}} \mathrm{dk}_{\mathrm{y}} \mathrm{dk}_{\mathrm{z}}$

respectively, where $e$ is the absolute charge of the electron, $m$ is the effective mass, $v_{x}$ the $x$ component of the electron velocity $\vec{v}, W_{F}$ the Fermi energy and $\vec{k}\left(k_{x}, k_{y}, k_{z}\right)$ the wave vector, and $\mathrm{f}^{1}$ the standard deviation from the equilibrium Fermi function. In the framework of the Cottey model, the deviation $\mathrm{f}^{1}$ is just written as

$\mathrm{f}^{1}=\frac{\mathrm{e}}{\hbar} \tau(\theta) \frac{\partial \mathrm{f}^{\mathrm{o}}}{\partial \mathrm{k}_{\mathrm{x}}}\left\{\mathrm{E}_{\mathrm{x}}+\frac{1}{\mathrm{e}} \frac{\mathrm{W}-\mathrm{W}_{\mathrm{F}}}{\mathrm{T}} \frac{\partial \mathrm{T}}{\partial \mathrm{x}}\right\}$

where $\tau(\theta)$ is the total relaxation time which describes the simultaneous background scattering and electronic scattering at the external rough surfaces.

Here it should be pointed out that to give the problem more tractable the calculations are performed in the free electron case for which the electron energy is simply given by

$\mathrm{W}=\frac{1}{2} \mathrm{~m}_{\mathrm{e}} \mathrm{v}^{2}=\frac{\hbar^{2} \mathrm{k}^{2}}{2 \mathrm{~m}_{\mathrm{e}}}$ 
so that for free electrons a constant energy surface of area $A$ satisfies the following relation $^{1}$

$\left[\frac{\partial \ln \mathscr{A}}{\partial \ln \mathrm{W}}\right]_{\mathrm{w}=\mathrm{w}_{\mathrm{F}}}=1$

Moreover we assume that for the free electron system the background relaxation time $\tau_{\mathrm{o}}$ is a function of energy only

$\tau_{\mathrm{o}}=\mathrm{b}_{\mathrm{o}} \mathrm{W}^{\mathrm{m}}$

where $b_{o}$ is a constant and $\mathrm{m}$ a number. ${ }^{15}$

To evaluate the current density $\mathrm{J}_{\mathrm{x}}$ we now need to express the total relaxation time. In terms of the Soffer-Cottey model (SC model) $\tau(\theta)$ can be written as ${ }^{8}$

$\tau(\theta)=\tau_{0}\left\{1+\mathrm{A}|\cos \theta| \cos ^{2} \theta\right\}^{-1}$

with

$\mathrm{A}=\left(\frac{4 \pi \mathrm{r}}{\lambda_{\mathrm{c}}}\right)^{2} \frac{\lambda_{\mathrm{o}}}{\mathrm{d}}=\mathrm{A}_{\mathrm{o}} \cdot \lambda_{\mathrm{o}}$

where $d$ is the film thickness. The mean free path $\lambda_{o}$ of all the free electrons can be expressed in terms of $\mathrm{W}$ :

$\lambda_{\mathrm{o}}=\left(\frac{2}{\mathrm{~m}_{\mathrm{e}}}\right)^{1 / 2} \mathrm{~b}_{\mathrm{o}} \mathrm{W}^{\mathrm{m}+1 / 2}$

where $\mathrm{m}_{\mathrm{e}}$ is the effective mass of free electrons.

Substituting Eqs. (8) and (4) into Eq. (2), introducing polar coordinates (k, $\theta, \Psi)$ in the $\overrightarrow{\mathrm{k}}$-space with $\mathrm{k}_{\mathrm{z}}=\mathrm{k} \cos \theta$, carrying our the integration over $\Psi$ and finally changing the integral with respect to $\mathrm{k}$ to one of $\mathrm{W}$ by means of Eq. (5), we have for the current density

$$
\begin{aligned}
J_{x}= & -\frac{4 \pi e^{2}}{h^{3}} m_{e} \int_{0}^{\infty} \int_{0}^{\pi} W \frac{\partial f^{\circ}}{\partial W}\left(E_{x}+\frac{1}{e} \frac{W-W_{F}}{T} \frac{\partial T}{\partial x}\right) \\
& \times \frac{\sin ^{3} \theta}{\frac{1}{\lambda_{o}(W)}+A_{o}|\cos \theta| \cos ^{2} \theta} d W d \theta
\end{aligned}
$$

If we now define

$$
K_{n f}^{*}=-\int_{0}^{\infty} \int_{0}^{\pi}\left(W-W_{F}\right)^{n} F_{f}(W, \theta) \frac{\partial f^{o}}{\partial W} d W d \theta
$$


where

$F_{f}(W, \theta)=\frac{W \cdot \sin ^{3} \theta}{\frac{1}{\lambda_{o}(W)}+A_{o}|\cos \theta| \cos ^{2} \theta}$

Eq. (11) simplifies to

$J_{x}=\frac{4 \pi e^{2}}{h^{3}} m_{e}\left\{E_{x} K_{o f}^{*}+\frac{1}{e T} \frac{\partial T}{\partial x} K_{1 f}^{*}\right\}$

The heat flux density may be obtained in a similar manner; using the integrals $\mathrm{K}_{\mathrm{nf}}^{*}$ gives

$u_{x}=-\frac{4 \pi e}{h^{3}} m_{e}\left\{E_{x} K_{1 f}^{*}+\frac{1}{e T} \frac{\partial T}{\partial x} K_{2 f}^{*}\right\}$

It remains to evaluate the integrals of $\mathrm{K}_{\mathrm{of}}^{*}, \mathrm{~K}_{1 \mathrm{f}}^{*}$ and $\mathrm{K}_{2 \mathrm{f}}^{*}$. This can be done by using the general expansion theorem in the region about $\mathrm{W}_{\mathrm{F}}{ }^{14}$

$-\int \mathrm{G}(\mathrm{W}) \frac{\partial f^{o}}{\partial \mathrm{W}} \mathrm{dW}=\mathrm{G}\left(\mathrm{W}_{\mathrm{F}}\right)+\frac{1}{6}\left(\pi \mathrm{k}_{\mathrm{B}} \mathrm{T}\right)^{2}\left\{\frac{\partial^{2} \mathrm{G}(\mathrm{W})}{\partial \mathrm{W}^{2}}\right\}_{\mathrm{W}=\mathrm{W}_{\mathrm{F}}}+\ldots$

where $\mathrm{k}_{\mathrm{B}}$ is the Boltzmann constant.

Applying the expansion theorem to the integral $\mathrm{K}_{\mathrm{of}}^{*}$ the second term in Eq. (16) is small compared with the first so that $\mathrm{K}_{\mathrm{of}}^{*}$ reduces to

$\mathrm{K}_{\mathrm{of}}^{*}=\mathrm{W}_{\mathrm{F}} \lambda_{\mathrm{o}}\left(\mathrm{W}_{\mathrm{F}}\right) \mathrm{I}_{1}$

with

$I_{1}=\int_{0}^{\pi} \frac{\sin ^{3} \theta}{1+A\left(W_{F}\right)|\cos \theta| \cos ^{2} \theta} d \theta$

Retaining the second term only the result for the integral $\mathrm{K}_{1 \mathrm{f}}^{*}$ is found to be

$\mathrm{K}_{1 \mathrm{f}}^{*}=\frac{\left(\pi \mathrm{k}_{\mathrm{B}} \mathrm{T}\right)^{2}}{3} \lambda_{\mathrm{o}}\left(\mathrm{W}_{\mathrm{F}}\right)\left\{\mathrm{I}_{1}+\left(\mathrm{m}+\frac{1}{2}\right) \mathrm{I}_{2}\right\}$

where

$I_{2}=\int_{0}^{\pi} \frac{\sin ^{3} \theta}{\left\{1+A\left(W_{F}\right)|\cos \theta| \cos ^{2} \theta\right\}^{2}} d \theta$

Similarly using Eq. (16), $\mathrm{K}_{2 \mathrm{f}}^{*}$ is quickly integrated and gives:

$\mathrm{K}_{2 \mathrm{f}}=\frac{\left(\pi \mathrm{k}_{\mathrm{B}} \mathrm{T}\right)^{2}}{3} \mathrm{~W}_{\mathrm{F}} \lambda_{\mathrm{o}}\left(\mathrm{W}_{\mathrm{F}}\right) \mathrm{I}_{1}$ 
We turn now to the transport coefficients $S_{\mathrm{f}}$ and $\mathscr{C}_{\mathrm{f}}$ and we start from the definition of the absolute thermopower and of the thermal conductivity which for the geometry of the model can be given by the respective general macroscopic relation:

$\left.E_{x}=S_{f} \frac{\partial T}{\partial x}\right]_{J_{x}=0}$

and

$\left.u_{\mathrm{x}}=-\mathscr{C}_{\mathrm{f}} \frac{\partial \mathrm{T}}{\partial \mathrm{x}}\right]_{\mathrm{J}=0}$

Applying Eq. (22) to Eq. (14) the absolute thermopower of the metal film is then

$S_{\mathrm{f}}=-\frac{1}{\mathrm{eT}} \frac{\mathrm{K}_{1 \mathrm{f}}^{*}}{\mathrm{~K}_{\mathrm{of}}^{*}}$

Combining Eqs. (15) and (23) the thermal conductivity then may be simply expressed as

$\mathscr{b}_{\mathrm{f}}=\frac{4 \pi \mathrm{m}_{\mathrm{e}}}{\mathrm{h}^{3}} \cdot \frac{1}{\mathrm{~T}}\left\{\mathrm{~K}_{2 \mathrm{f}}^{*}-\frac{\mathrm{K}_{1 \mathrm{f}}^{*}}{\mathrm{~K}_{\mathrm{of}}^{*}}\right\}$

\section{ANALYTICAL EXPRESSIONS FOR THE TRANSPORT COEFFICIENTS}

We now proceed with the integration of $I_{1}$ and $I_{2}$. Integral $I_{1}$ resembles to the integral which reflects the effect of the external scattering of conduction electrons on the film conductivity ${ }^{8}$ Then $I_{1}$ is readily found to be written as

$\mathrm{I}_{1}=\frac{2}{3} \mathscr{F}(\kappa)$

with

$\mathscr{F}(\kappa)=\kappa G(\kappa)-\kappa^{3} \ln \left(1+\frac{1}{\kappa^{3}}\right)$

$\mathrm{G}(\kappa)=\frac{1}{2} \ln \frac{(\kappa+1)^{2}}{\kappa^{2}-\kappa+1}+\sqrt{3} \operatorname{Tan}^{-1}\left(\frac{2-\kappa}{\sqrt{3} \kappa}\right)-\sqrt{3} \operatorname{Tan}^{-1}\left(-\frac{1}{\sqrt{3}}\right)$

and

$\kappa=A^{-1 / 3}$ 
Rearranging integral $\mathrm{I}_{2}$ and integrating by part leads to

$\mathrm{I}_{2}=\frac{2}{3}\left(\frac{2}{3} \mathrm{k} \cdot \mathrm{G}(\mathrm{k})\right)$

Introducing Eqs. (26) and (30) into Eqs. (17) and (19) gives after simple mathematical arrangements the film thermopower as

$\mathrm{S}_{\mathrm{f}}=\frac{\left(\pi \mathrm{k}_{\mathrm{B}}\right)^{2} \mathrm{~T}}{3 \mathrm{e} \mathrm{W}_{\mathrm{F}}}\left[1+\left(\mathrm{m}+\frac{1}{2}\right) \frac{2}{3}\left(1+\frac{\mathrm{\kappa}^{3} \ln \left(1+1 / \mathrm{\kappa}^{3}\right)}{\mathscr{F}(\mathrm{\kappa})}\right)\right]$

It is convenient to pause here and to consider the theoretical expression for the temperature coefficient of resistivity, $\beta_{\mathrm{f}}$, in the case of electron scattering at rough surfaces which was derived ${ }^{12}$ neglecting the thermal expansion of the film thickness with respect to that of the bulk mean free path, i.e.

$\beta_{\mathrm{f}} / \beta_{\mathrm{o}}=\frac{2}{3}\left(1+\frac{\kappa^{3} \ln \left(1+1 / \kappa^{3}\right)}{\mathscr{F}(\kappa)}\right)$

where the subscript o denote the bulk material. Hence for free electron the thermoelectric power can be simply expressed in terms of t.c.r. ratio, $\beta_{\mathrm{f}} / \beta_{\mathrm{o}}$,

$S_{\mathrm{f}}=-\mathrm{s}\left(1+\left(\mathrm{m}+\frac{1}{2}\right) \frac{\beta_{\mathrm{f}}}{\beta_{\mathrm{o}}}\right)$

with

$\mathrm{s}=\frac{\left(\pi \mathrm{k}_{\mathrm{b}}\right)^{2} \mathrm{~T}}{3 \mathrm{e} \mathrm{W}_{\mathrm{F}}}$

Moreover the most general expression for the diffusion thermopower in metal films is given by ${ }^{1,15}$

$\left.S_{\mathrm{f}}=-\mathrm{s} \frac{\partial \ln \sigma_{\mathrm{f}}}{\partial \ln \mathrm{W}}\right]_{\mathrm{W}=\mathrm{w}_{\mathrm{F}}}$

where $\sigma_{\mathrm{f}}$ is the electrical conductivity of the metal film. In the case of spherical Fermi surface with isotropic background relaxation time $\tau_{\mathrm{o}}$ the conductivity $\sigma_{\mathrm{f}}$ can be written in the form ${ }^{8}$ :

$\sigma_{\mathrm{f}} \sim \mathscr{A} \cdot \lambda_{\mathrm{o}} \cdot \mathrm{H}\left(\lambda_{\mathrm{o}}\right)$ 
Defining the energy-dependent terms

$\left.\left.\mathrm{U}=\frac{\partial \ln \lambda_{\mathrm{o}}}{\partial \ln \mathrm{W}}\right]_{\mathrm{W}=\mathrm{w}_{\mathrm{F}}}, \mathrm{V}=\frac{\partial \ln \mathscr{A}}{\partial \ln \mathrm{W}}\right]_{\mathrm{W}=\mathrm{w}_{\mathrm{F}}}$

differentiation of Eq. (36) gives

$S_{\mathrm{f}}=-s\left\{\mathrm{~V}+\mathrm{U}\left(\beta_{\mathrm{f}} / \beta_{\mathrm{o}}\right)\right\}$

If the electrons are free $\mathrm{V}$ and $\mathrm{U}$ reduce to 1 and $\mathrm{m}+\frac{1}{2}$ respectively. Thus comparing Eqs. (31) and (38) we see that a more general expression of the form

$S_{\mathrm{f}}=-\mathrm{s}\left\{\mathrm{V}+\mathrm{U} \frac{2}{3}\left(1+\frac{\kappa^{3} \ln (1+1 / \kappa)}{\mathscr{F}_{(}(\kappa)}\right)\right\}$

is appropriate for films of metals with nearly spherical Fermi surface.

Starting from the general expression of the thermal conductivity, $\mathscr{C}_{\mathrm{f}}$, we have for the thermal conductivity:

$\mathscr{b}_{\mathrm{f}}=\mathrm{B}\left[\frac{2}{3} \mathscr{F}(\kappa)-\mathrm{c} \frac{\left[\mathscr{F}_{(\kappa)}+\frac{2}{3}\left(\mathrm{~m}+\frac{1}{2}\right) \kappa \mathrm{G}(\mathrm{\kappa})\right]^{2}}{\mathscr{F}_{(\kappa)}}\right]$

where $\mathrm{B}$ and $\mathrm{C}$ are two constants given by

$\mathrm{B}=\frac{4 \pi^{3} \mathrm{k}_{\mathrm{B}}^{2} \mathrm{~m}_{\mathrm{e}} \lambda_{\mathrm{o}} \mathrm{W}_{\mathrm{F}}}{3 \mathrm{~h}^{3}} \cdot \mathrm{T}$

$c=\frac{\left(\pi \mathrm{k}_{\mathrm{B}} \mathrm{T}\right)^{2}}{3 \mathrm{~W}_{\mathrm{F}}^{2}}$

We shown that to the first order in T Eq. (40) yields

$\mathscr{C}_{\mathrm{f}}=\frac{2}{3} \mathrm{~B} \mathscr{F}(\kappa)$

Here it must be kept in mind that the film conductivity, $\sigma_{\mathrm{f}}$, is just ${ }^{8}$

$\sigma_{\mathrm{f}}=\frac{\sigma_{\mathrm{o}}}{2} \cdot \mathscr{F}(\kappa)$

so that Eq. (43) leads directly to the law of Wiedemann-Franz $z^{15,16}$

$\mathscr{C}_{\mathrm{f}} / \sigma_{\mathrm{f}}=\frac{\pi^{2} \mathrm{k}_{\mathrm{B}}^{2} \mathrm{~T}}{3 \mathrm{e}^{2}}=\mathscr{L}$ 


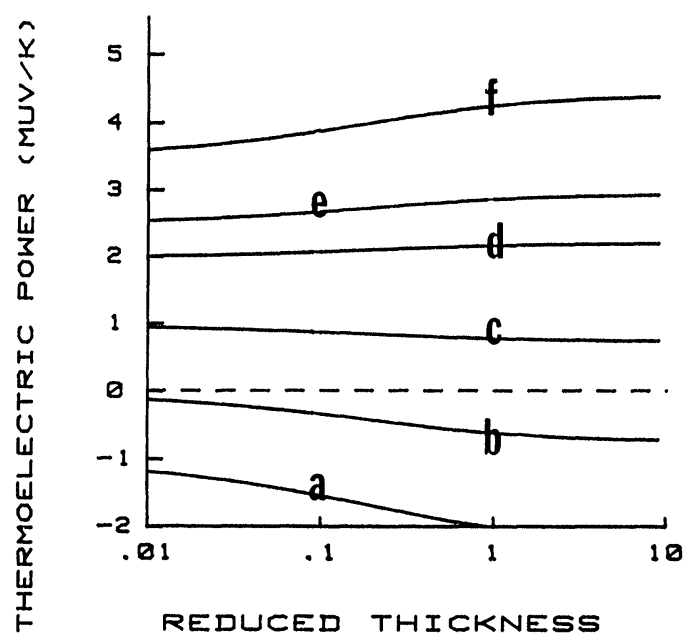

FIGURE 1 Plots of $S_{\mathrm{f}}$ against $\mathrm{k}$ for $\mathrm{r} / \lambda_{\mathrm{c}}=0.06, \mathrm{~W}_{\mathrm{F}}=5 \mathrm{eV}$ and for different values of $\mathrm{m}$. Curves $\mathrm{a}, \mathrm{b}, \mathrm{c}$, $\mathrm{d}, \mathrm{e}, \mathrm{f}$ are for $\mathrm{m}=-3,-2,-1,0,0.5$ and 1.5 respectively.

which remains valid for all metal films provided $\mathrm{k}_{\mathrm{B}} \mathrm{T} / \mathrm{W}_{\mathrm{F}}$ is small. The deviation from the constancy of the Lorentz number, $\mathscr{L}$, (referred as D.L.N. hereafter) can then be conveniently expressed as:

D.L.N. $=c\left(\mathscr{F}(\kappa)+\frac{2}{3}\left(\mathrm{~m}+\frac{1}{2}\right) \kappa \mathrm{G}(\kappa)\right)^{2}[\mathscr{F}(\kappa)]^{-1}$

\section{THEORETICAL RESULTS}

\section{The Reduced Thermoelectric Power}

For a sake of simplicity, the results presented in this section are essentially concerned with the free electron case for which the thermopower, $S_{o}$, of the bulk material is given by

$\mathrm{S}_{\mathrm{o}}=-\mathrm{s}\left(\mathrm{m}+\frac{3}{2}\right)=-\mathrm{s}(\mathrm{V}+\mathrm{U})$

Equation (31) can be evaluated numerically with the aid of a pocket calculator but for convenience the theoretical curves illustrating this section are drawn using a microcomputer.

In our discussion of thermopowers of metal films we are inevitably forced to discuss the influence of the constant $m$ on the reduced thickness, $k=d / \lambda_{o}$, or on the reduced roughness, $r / \lambda_{c}$, dependence of the thermopower ratio, $S_{F} / S_{o}$. For this purpose it seems suitable to examine (Fig. 1) the theoretical variations in the thermopower $S_{F}$ of a mod- 


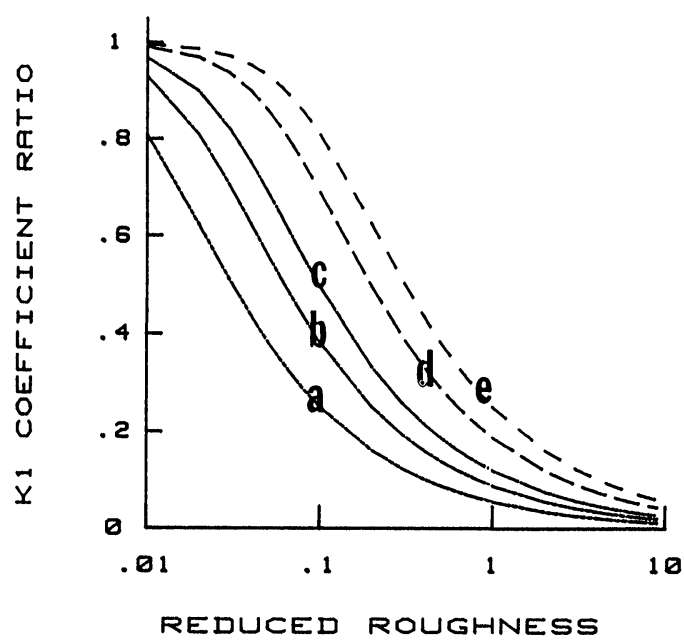

FIGURE 2 Plots of the ratio $\mathrm{K}_{1 \mathrm{f}}^{*} / \mathrm{K}_{1 \mathrm{o}}^{*}$ against $\mathrm{r} / \lambda_{\mathrm{c}}$ for $\mathrm{m}=1.5, \mathrm{~W}_{\mathrm{F}}=5 \mathrm{eV}$ and for different values of $\mathrm{k}$. Curves $\mathrm{a}, \mathrm{b}, \mathrm{c}, \mathrm{d}, \mathrm{e}$ are for $\mathrm{k}=0.01,0.04,0.1,0.4$ and 1 respectively.

erately rough film $\left(r / \lambda_{c}=0.06\right)$ for different values of the constant $\mathrm{m}$. Figure 1 undoubtedly indicates, except in an intermediate range of values of $\mathrm{m}$ for which $-1.5<$ $\mathrm{m}<-0.5$, that the absolute magnitude of the film thermopower increases with increasing values of the reduced thickness as frequently observed in experiments. ${ }^{17-26}$ In contrast, in the intermediate region the magnitude of $S_{\mathrm{F}}$ falls monotonously to the bulk one.

Taking into account this remark in Figs. 2 and 3 we have plotted the theoretical variations in the reduced coefficient, $K_{1 \mathrm{f}}^{*} / \mathrm{K}_{1 \mathrm{o}}^{*}$, as function of the reduced roughness, $r / \lambda_{\mathrm{c}}$, for these two different ranges of $m$ values, respectively. Several points arising from these figures merit some comments:

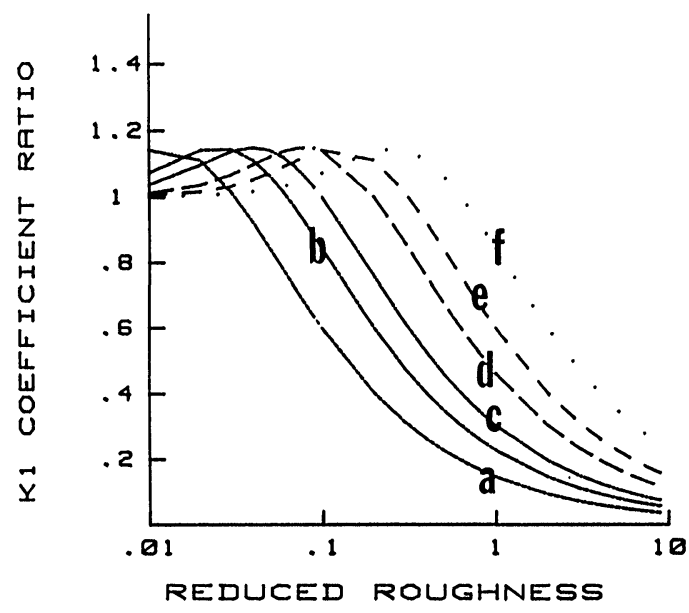

FIGURE 3 Plots of the ratio $\mathrm{K}_{1 \mathrm{f}}^{*} / \mathrm{K}_{1 \text { o }}^{*}$ against $\mathrm{r} / \lambda_{\mathrm{c}}$ for $\mathrm{m}=-1.25, \mathrm{~W}_{\mathrm{F}}=5 \mathrm{eV}$ and for different values of k. Curves a, b, c, d, e, f are for $\mathrm{k}=0.01,0.04,0.1,0.4,1$ and 4 respectively. 
TABLE I

Values of the coefficient $\mathrm{K}_{1}^{*}$ ratio, $\mathrm{K}_{1 \mathrm{f}}^{*} / \mathrm{K}_{10}^{*}$ as evaluated in terms of the Cottey model and the $\mathrm{SC}$ model. The energetic parameter $\mathrm{m}$ is taken equal to $3 / 2$.

\begin{tabular}{lccccccc}
\hline & \multicolumn{3}{c}{ Cottey model } & & \multicolumn{3}{c}{ Present model } \\
\cline { 2 - 3 } \cline { 6 - 7 } $\mathrm{k}$ & $\mathrm{p}=0.4$ & $\mathrm{p}=0.6$ & $\mathrm{p}=0.9$ & $\mathrm{r} / \lambda_{\mathrm{c}}=0.1$ & $\mathrm{r} / \lambda_{\mathrm{c}}=0.06$ & $\mathrm{r} / \lambda_{\mathrm{c}}=0.02$ \\
\hline 0.04 & 0.0996 & 0.1541 & 0.4194 & 0.3840 & 0.5105 & 0.8100 \\
0.1 & 0.1950 & 0.2868 & 0.6250 & & 0.4965 & 0.6386 & 0.8998 \\
0.4 & 0.4502 & 0.5824 & 0.8621 & & 0.6903 & 0.8222 & 0.9696 \\
1 & 0.6549 & 0.7674 & 0.9389 & & 0.8100 & 0.9078 & 0.9873 \\
4 & 0.8775 & 0.9271 & 0.9838 & & 0.9315 & 0.9724 & 0.9967 \\
\hline
\end{tabular}

1. As the energetic constant $m$ takes values outside the previously defined intermediate range, the $\mathrm{K}_{1}^{*}$ coefficient ratio exhibits a roughness dependence in accord, at first sight, with those related to other transport parameters (electrical conductivity, t.c.r. . . . ${ }^{8,12}$ : with increasing surface roughness the coefficient $\mathrm{K}_{1}^{*}$ ratio falls rapidly to zero even for relatively thick films $(k>1)$ (Fig. 2).

2. Concerning our attention to the thickness dependence (Fig. 2) it appears that in reality the incorporation of both the surface roughness and the angular dependence in the transport parameter results in a decrease of the overall size effect as compared with the predictions of the Cottey model. ${ }^{1}$ To provide more information on the difference in theoretical results as evaluated from the present model on the one hand and from the Cottey model on the other hand, theoretical values of the coefficient $\mathrm{K}_{1}^{*}$ ratio are displayed in Table I for the respective range of applicability of the two models (i.e., $p>0.4$ and $\mathrm{k}>0.04$ for the Cottey model, ${ }^{27} \mathrm{r} / \lambda_{\mathrm{c}}<0.4$ for the SC model). ${ }^{8}$ From table I it clearly appears that the previous main conclusion on the overall size effect is confirmed.

3 . In the intermediate range the ratio $\mathrm{K}_{1 \mathrm{f}}^{*} / \mathrm{K}_{1 \mathrm{o}}^{*}$ exhibits a more remarkable behavior. For a given film thickness we see a peak in the variations of $K_{1 \mathrm{f}}^{*} / \mathrm{K}_{1 \mathrm{o}}^{*}$ with $\mathrm{r} / \boldsymbol{\lambda}_{\mathrm{c}}$ (Figure $3)$. As the film becomes thinner and thinner the peak appears for smoother and smoother film surfaces. This behavior seems to be characteristic to the SC model which includes the angular dependence of the specularity parameter. Effectively, theoretical classical models derived in the framework of the F.S. theory ${ }^{28,29}$ or the Cottey theory predict a continuous and monotoneous decrease in $\mathrm{K}_{1 \mathrm{f}}^{*} / \mathrm{K}_{1 \mathrm{o}}^{*}$ as the electron scattering at external surfaces changes from totally specular to increasingly diffuse.

4. As expected the size effect vanishes when the film has zero roughness (specular scattering) or when the film thickness tends to infinity.

It is now interesting to see more precisely what happens in the variation of the thermopower ratio, $S_{\mathrm{f}} / \mathrm{S}_{\mathrm{o}}$, against the reduced thickness, $\mathrm{k}$, for different values of $\mathrm{m}$. Figure 4 shows that if $\mathrm{m}$ lies in the range where $\mathrm{m}>0$ or $\mathrm{m}<-1.5$, as $\mathrm{k}$ increases, $\mathrm{S}_{\mathrm{f}}$ rises to reach the value for the bulk specimen in crude accord with classical models ${ }^{28,29}$ for the size effect. For $\mathrm{m}=-1.25$ (Fig. 5) never the film thermopower reaches a maximum but there is a marked fall of $S_{f} / S_{o}$ to unity with increasing $k$. Hence if we look to Eq. (24) we see that the apparent effect of the variation in the coefficient $\mathrm{K}_{\mathrm{of}}^{*}$ with $\mathrm{k}$ is to give ineffective the $\mathrm{K}_{1 \mathrm{f}}^{*}$ maximum which is not very sharp so that the thermopower shows 


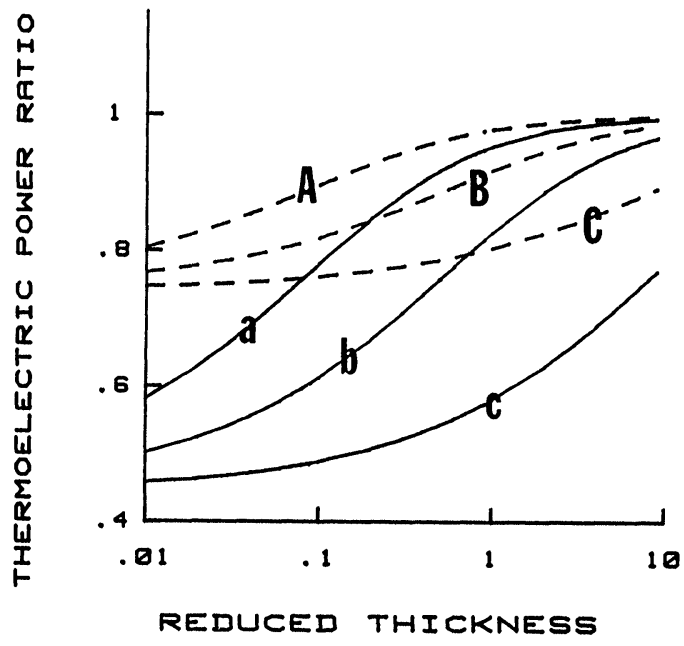

FIGURE 4 Plots of the ratio $S_{F} / S_{o}$ against $k$ for $W_{F}=5 \mathrm{eV}$ and for different values of $\mathrm{m}$ and $\mathrm{r} / \lambda_{\mathrm{c}}$. Curves $\mathrm{A}, \mathrm{B}, \mathrm{C}$ are for $\mathrm{m}=-3$ and for $\mathrm{r} / \lambda_{\mathrm{c}}=0.04,0.1$ and 0.4 respectively. Curves $\mathrm{a}, \mathrm{b}, \mathrm{c}$ are for $\mathrm{m}=3$ and for $\mathrm{r} / \lambda_{\mathrm{c}}=0.04,0.1$ and 0.4 respectively.

a monotonous decrease over a wide range of $\mathrm{k}$. This behavior remains at first sight quite similar to the behavior predicted by the Cottey model even if in the Cottey model the overall size effect is again more accentuated than in the SC model (Table II).

\section{The reduced thermal conductivity}

In Fig. 6 the thermal conductivity ratio, $\mathscr{C}_{\mathrm{f}} / \mathscr{C}_{\mathrm{o}}$, is plotted versus $\mathrm{k}$ for different values of the reduced roughness. In this case the value of the r.m.s. surface roughness plays an

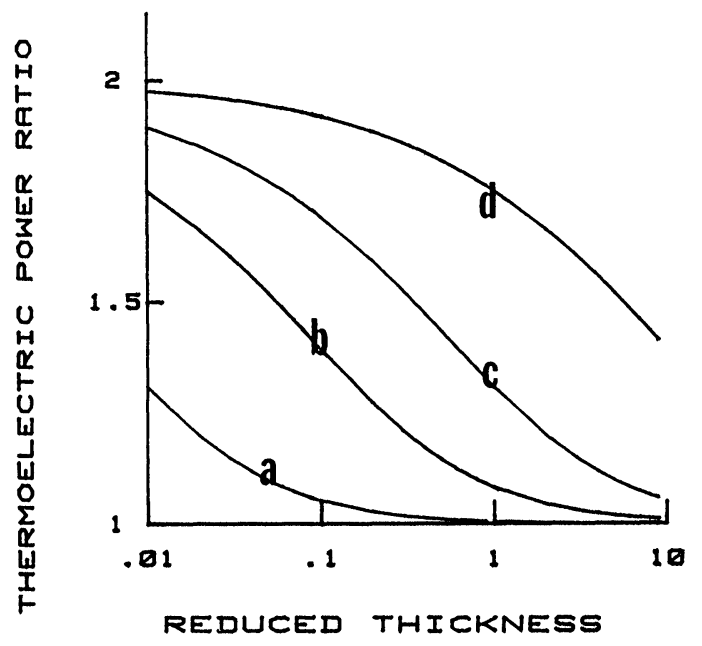

FIGURE 5 Plots of the ratio $S_{F} / S_{o}$ against $k$ for $W_{F}=5 \mathrm{eV}, \mathrm{m}=-1.25$ and different values of $\mathrm{r} / \lambda_{\mathrm{c}}$. Curves $\mathrm{a}, \mathrm{b}, \mathrm{c}, \mathrm{d}$ are for $\mathrm{r} / \lambda_{\mathrm{c}} 0.01,0.04,0.1$ and 0.4 respectively. 
TABLE II

Values of the thermopower ratio, $\mathrm{S}_{\mathrm{f}} \mathrm{S}_{\mathrm{o}}$, as evaluated in terms of the Cottey model and the SC model. The energetic parameter $\mathrm{m}$ is taken equal to -1.25 .

\begin{tabular}{lccccccc}
\hline & \multicolumn{3}{c}{ Cottey model } & & \multicolumn{3}{c}{ Present model } \\
\cline { 2 - 3 } \cline { 6 - 7 } $\mathrm{k}$ & $\mathrm{p}=0.4$ & $\mathrm{p}=0.6$ & $\mathrm{p}=0.9$ & $\mathrm{r} / \lambda_{\mathrm{c}}=0.1$ & $\mathrm{r} / \lambda_{\mathrm{c}}=0.06$ & $\mathrm{r} / \lambda_{\mathrm{c}}=0.02$ \\
\hline 0.04 & 2.9769 & 2.7977 & 2.1321 & 1.7993 & 1.6817 & 1.3104 \\
0.1 & 2.6786 & 2.4391 & 1.7046 & 1.6958 & 1.5394 & 1.1714 \\
0.4 & 2.0651 & 1.7898 & 1.2514 & 1.4752 & 1.2924 & 1.0539 \\
1 & 1.6454 & 1.4288 & 1.1105 & & 1.3104 & 1.1585 & 1.0228 \\
4 & 1.2230 & 1.1321 & 1.0291 & 1.1191 & 1.0489 & 1.0059 \\
\hline
\end{tabular}

important role, a small value resulting in a small overall size effect and vice versa. In reality the ratio of film to bulk thermal conductivity in metals behaves in the same manner as the electrical conductivity. As a consequence the D.L.N. will certainly takes negligible values in the $k$ and $r / \lambda_{c}$ ranges under consideration here. The values for the D.L.N. evaluated from Eq. (46) are listed in Table III. We have calculated the D.L.N. for a metal film with a Fermi energy of $5 \mathrm{eV}$, in this way the main conclusions drawn in this section apply to noble metals, $\mathrm{Cu}, \mathrm{Ag}$ and $\mathrm{Au}$. As roughening of the external surfaces causes a marked decrease in the D.L.N. the Lorentz number $\mathscr{L}$ can be regarded as independent of size effects. A situation which is agreement with what we find $^{29}$ starting from the classical models.

\section{DISCUSSION AND CONCLUSION}

If we turn our attention to the variations of the transport parameters with film thickness it clearly appears that the angular dependence and the r.m.s. surface roughness diminish

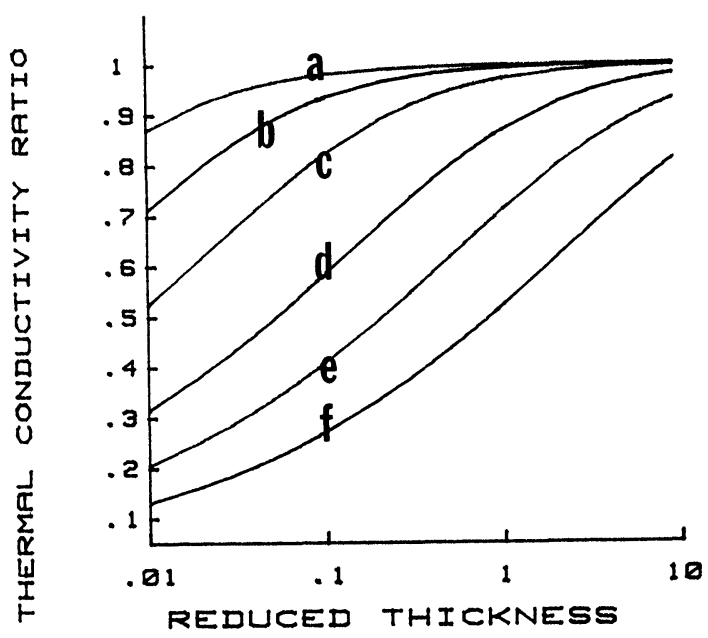

FIGURE 6 Plots of the ratio $\mathscr{C}_{\mathrm{f}} / \mathscr{C}_{\mathrm{o}}$ against $\mathrm{k}$ for $\mathrm{W}_{\mathrm{F}}=5 \mathrm{eV}, \mathrm{m}=1.5$ and for different values of $\mathrm{r} / \lambda_{\mathrm{c}}$. Curves $\mathrm{a}, \mathrm{b}, \mathrm{c}, \mathrm{d}, \mathrm{e}, \mathrm{f}$ are for $\mathrm{r} / \lambda_{\mathrm{c}}=0.01,0.02,0.04,0.1,0.2$ and 0.4 respectively. 
TABLE III

Theoretical values of $10^{3} \times$ D.L.N. assuming the energetic parameter $\mathrm{m}=3 / 2$.

\begin{tabular}{lccc}
\hline $\mathrm{k}$ & $\mathrm{r} / \lambda_{\mathrm{c}}=0.01$ & $\mathrm{r} / \lambda_{\mathrm{c}}=0.04$ & $\mathrm{r} / \lambda_{\mathrm{c}}=0.1$ \\
\hline 0.01 & 0.7988 & 0.3849 & 0.2136 \\
0.04 & 0.9606 & 0.5788 & 0.3346 \\
0.1 & 1.0148 & 0.7261 & 0.4446 \\
0.4 & 1.0474 & 0.9169 & 0.6540 \\
1 & 1.0545 & 0.9916 & 0.7988 \\
\hline
\end{tabular}

the size effect so that one can reasonably expect smaller size effect in the thermopower or in the thermal conductivity when we treat the thermal properties in terms of the SC model than we return to the Cottey formulation. Furthermore if we examine again Eq. (38) and if we remember that the SC model leads to a decrease in the overall size effect which is considerably more marked for the film t.c.r. ${ }^{12}$ than for the film conductivity one can conclude that transforming a smooth surface to a rough surface gives rise to large size effect only if the energetic parameter $m$ takes large positive or negative values. So the situation departs from the free electron case for which $\mathrm{m}=3 / 2$ and from the case of electron scattering by impurity atoms for which it is generally assumed $\mathrm{m}=-1 / 2 .{ }^{15}$ Moreover large negative values for $\mathrm{m}$ have been considered, until recently, as physically unreasonable values even if these anomalous have been frequently reported in literature. ${ }^{19-21,25}$ At this point of the discussion it should be noticed that here the calculations are performed using a single relaxation time $\tau_{\mathrm{o}}$ for the background electron scattering. But as films contain structural defects such as vacancies, dislocations and grain-boundaries which affect ${ }^{1}$ the thermopower one can believe that the energy dependence of the relaxation time or the mean free path will change strongly with a large concentration of defects. ${ }^{15,30}$ This problem is frequently interpreted ${ }^{1}$ in terms of the Nordheim-Gorter's rule ${ }^{15}$ assuming the validity of the Matthiessen's rule. It seems also convenient to discuss what happens when the energetic parameter $\mathrm{V}$ differs from that $(\mathrm{V}=1)$ for free electrons. Several authors $^{15,31,32}$ have pointed out that if distortion of the Fermi surface occurs due to additional electron scattering by impurities the energetic factor $\mathrm{V}$ could become negative. Moreover as suggested by Ziman ${ }^{14}$ noble metals with distorted Fermi surface can also have positive diffusion thermopower as the result of a decrease of the relaxation time with increase in energy which arises with increasing distortion of the Fermi surface.

It is well known that in thin films the concentration of structural defects is large enough, however we cannot neglect the presence of impurities frozen in the film. Owing to the preceding remarks this situation gives inevitably rise to a marked deviation in the value of the two energetic parameters $U$ and $V$ with respect to that measured for a perfect bulk specimen. In Table IV we list the result of measurements of $S_{\mathrm{f}}$ on films of $\mathrm{Au}, \mathrm{Ag}$ and $\mathrm{Cu}$. For some metals we observe a large scatter between results from different authors which can be attributed to differences in the preparation procedure and subsequent annealing process which certainly induce the formation of thin films whose final structure and chemical composition (concentration of impurity) differ notably. In these conditions changing the value of $U$ and/or $V$ we can certainly verify the adequation of the present model to describe the size effect in the thermopower provided in previous experiments the variation of $S_{f}$ with $k$ was found to be in closer agreement with the predictions of 
TABLE IV

Energetic parameter data as determined from measurement of the thermopower on metal films.

\begin{tabular}{lcl}
\hline Metal & $\mathrm{U}$ & $\mathrm{V}$ \\
\hline Au (17) & -0.6 & -0.8 \\
Au (33) & -0.6 & -0.7 \\
Au (34) & -0.5 & -1.05 \\
Ag (25) & 3 & -4.4 \\
Ag (35) & 1.15 & -2.32 \\
Cu (19) & -0.5 & -1.10 \\
Cu (21) & 0.7 & -2.1 \\
Cu (20) & -18.7 & $\ldots$ \\
\hline
\end{tabular}

the classical theory. Hence let us consider a few experimental works ${ }^{18,26,36}$ to discuss the validity of the present model.

First of all let us examine the measurements of $S_{f}$ on evaporated of $\mathrm{Cu}^{18}$ and $\mathrm{Ag}^{36}$ by Leonard and $\mathrm{Yu}$. The fact that moderate size effects at room temperature have been obtained in these $\mathrm{Cu}$ and $\mathrm{Ag}$ films up to $\mathrm{k}>4$ can be understood in terms of the SC model for rough surfaces even if a proper annealing can induce a mechanical reordering of the top surface of films. ${ }^{1,37-41}$ This argument is sustained by the observation of correlated size effect in the corresponding temperature coefficient of resistivity. Data obtained from measurements of the thermoelectric power of $\mathrm{Cu}$ and $\mathrm{Ag}$ films are reinterpreted in terms of the SC model [Eq. (39)] retaining for the energetic parameters $U$ and $V$ the values calculated by Leonard and $\mathrm{Yu}$. Theoretical values of the surface roughness are displayed in Table $\mathrm{V}$. In the calculation, in agreement with Leonard and $\mathrm{Yu}$, a value of $1.55 \mu \mathrm{V} / \mathrm{K}$ and $1.7 \mu \mathrm{V} / \mathrm{K}$ is used for the bulk thermopower of $\mathrm{Ag}$ and $\mathrm{Cu}$ respectively. The values of the r.m.s. surface roughness in the range $(0.33-0.18)$ are physically consistent and indicate that the observed variation in the thermopower can be understood in terms of the SC model. At this stage it seems interesting to note that for annealed $\mathrm{Cu}$ and $\mathrm{Ag}$ films the observed background thermopower does not depart very much from the bulk value cited in literature. Hence apparently the grain boundary scattering process does not play an important role when we turn to the size dependence of the thermopower the essence of which is essentially the electron scattering at external surfaces. Consequently a rough independence of $r / \lambda_{c}$ (copper films) or small changes in $r / \lambda_{c}$ (silver films)

TABLE V

Changes in the r.m.s. surface roughness, $r / \lambda_{c}$, with the reduced thickness $k$.

\begin{tabular}{|c|c|c|c|c|c|}
\hline \multicolumn{3}{|c|}{ Ag films (36) } & \multicolumn{3}{|c|}{$\begin{array}{c}\text { Cu films (18) } \\
\mathrm{U}=-0.21, \mathrm{~V}=-1.43, \lambda_{\circ}=380 \AA\end{array}$} \\
\hline $\mathrm{k}$ & $\Delta S(\mu \mathrm{V} / \mathrm{K})$ & $\mathrm{r} / \lambda_{\mathrm{c}}$ & $\mathrm{k}$ & $\Delta S(\mu \mathrm{V} / \mathrm{K})$ & $\mathrm{r} / \boldsymbol{\lambda}_{\mathrm{c}}$ \\
\hline 1.38 & 0.385 & 0.33 & 2.24 & -0.043 & 0.33 \\
\hline 2.10 & 0.305 & 0.27 & 2.33 & -0.0556 & 0.60 \\
\hline 2.42 & 0.265 & 0.24 & 2.67 & -0.0330 & 0.24 \\
\hline 2.70 & 0.260 & 0.24 & 4.52 & -0.0308 & 0.29 \\
\hline 3.35 & 0.180 & 0.19 & 6.20 & -0.0285 & 0.31 \\
\hline 5.24 & 0.125 & 0.18 & & & \\
\hline
\end{tabular}


TABLE VI

Variations in the r.m.s. surface roughness, $r / \lambda_{c}$, with the thickness, $d$, of polycrystalline tin films $\left(\mathrm{U}=1, \mathrm{~V}=2, \mathrm{~S}_{\mathrm{g}}=-1.65 \mu \mathrm{V} / \mathrm{K}\right.$, background m.f.p. $\left.\lambda_{\mathrm{g}} \simeq 550 \AA,(26)\right)$

\begin{tabular}{lllllr}
\hline $\mathrm{d}(\AA)$ & 500 & 1000 & 1900 & 3200 & 7000 \\
\hline $\mathrm{r} / \lambda_{\mathrm{c}}$ & 0.34 & 0.21 & 0.16 & 0.13 & 0.12 \\
$\mathrm{k}$ & 0.91 & 1.92 & 3.45 & 5.82 & 12.72 \\
\hline
\end{tabular}

with thickness agrees with the common idea that for almost epitaxial relatively thick films (d > $800 \AA$ ) a complete annealing process leads to similar geometrical properties of the external surfaces whatever the film thickness.

Also Das and Mohanty observed the thermopower of polycrystalline tin films ${ }^{26}$ to increase slowly with film thickness for $\mathrm{k}>2$. They analysed their data in terms of the effective mean free path model proposed several years ago by Tellier $^{42,43}$ to find an appropriate value of $-1.65 \mu \mathrm{V} / \mathrm{K}$ for the grain-boundary thermopower $\mathrm{Sg}$. Adopting this value for the background thermopower and assuming, as suggested by Das and Mohanty, that the energetic parameters $U$ and $V$ take values from the free electron model we are able to try to reconsider the size effect in these polycrystalline tin films in the framework of the SC model. The experimental values obtained for $\mathrm{k}$ and $\mathrm{r} / \lambda_{\mathrm{c}}$ on the annealed Tin films are listed in Table VI. Here again the maximum of $r / \lambda_{c}$ which occurs for thinner films does not reach 0.4 in accord with the maximum value obtained for silver films. Enhancement of the thickness variation of the r.m.s. surface roughness may be attributed to the presence of crystallites whose average size varies with the film thickness. Effectively one would expect the top surface becomes smoother with increasing grain size. Moreover it must be pointed out that tentative ${ }^{44}$ to fit previously published data on the thickness dependence of the Hall coefficient for annealed polycrystalline copper films revealed that the r.m.s. surface roughness has a behavior similar to the variation in $r / \lambda_{c}$ shown in table VI. In particular the limiting values $(0.25,0.12)$ of $r / \lambda_{c}$ are closer in magnitude with those observed for polycrystalline tin.

Before concluding it should be pointed out that the Soffer model is based on the theory of scattering of electromagnetic waves from rough surfaces proposed several years ago by Beckmann ${ }^{46}$ and that Eq. (1) holds only in the case of no correlation. Hence the validity of the model is only good if the radius of curvature of the surface irregularities is large compared with the de Broglie wavelength which for metals remains in most cases smaller than $5 \AA$. Since this implies that the roughness does not include sharp edges the S.C. model seems to be essentially concerned with surface asperities of the atomic scale such as, for example, atomic steps. Our experimental values of $r / \lambda_{c}$ (Table VI) agree well with this physical requirement.

With these few examples we see that the present model can be made to yield a fair agreement provided the concentration of frozen-in structural defect remains small. In particular it becomes possible to follow roughly the changes in the r.m.s. surface roughness with the film thickness. Physically reasonable values of $r / \lambda c$ are thus determined for $\mathrm{Cu}$, $\mathrm{Ag}$ and Sn films. But care must be taken that structural defects or impurities can always play a significant role in determining the size effect so that a consistent interpretation follows only from systematic studies for obtaining quantitative information on the nature of impurity and on the morphology. 


\section{REFERENCES}

1. C.R. Tellier and A.J. Tosser, Size Effects in Thin Films, (Elsevier, Amsterdam, 1982).

2. K. Fuchs, Proc. Cambridge Phil. Soc., 34 (1938) 100

3. E.H. Sondheimer, Adv. Phys., 1 (1952) 1

4. J.M. Ziman, Electrons and Phonons, (Oxford University Press, London, 1962, Chapter IX)

5. S. Soffer, J. Appl. Phys., 38 (1967) 1710

6. K.M. Leung, Phys. Rev. B., 30 (1984) 647

7. J.R. Sambles and K.C. Elsom, J. Phys. D : Appl. Phys., 10 (1977) 1329

8. C.R. Tellier, J. Mater. Sci. Lett., 3 (1984) 464

9. T.W. Preistand and J.R. Sambles, J. Phys. F : Met. Phys., 16 (1986) 2119

10. C.R. Tellier, J. Mater. Sci., 23 (1988) 1464

11. A.A. Cottey, Thin Solid Films, 1 (1967-68) 297

12. C.R. Tellier, J. Mater. Sci., 22 (1987) 2043

13. R.J.M. van Vucht, H. van Kempen and P. Wyder, Rep. Prog. Phys., 48 (1985) 853.

14. F.S. Blatt, Physics of Electronic Conduction in Solids, (Mc Graw Hill, New York, 1968)

15. R.D. Barnard, Thermoelectricity in Metals and Alloys, (Taylor and Francis, London, 1972) chapter 3

16. L. Ouarbya and A.J. Tosser, J. Mater. Sci., 16 (1981) 2287

17. S.F. Lin and W.F. Leonard, J. Appl. Phys., 42 (1971) 3634

18. W.F. Leonard and H-Y Yu, J. Appl. Phys., 44 (1973) 5320

19. V.V.R. Narasimha Rao, S. Mohan and P. Jayarama Reddy, J. Phys. D: Appl. Phys., 9 (1976) 89

20. K.L. Chopra, S.K. Bahl and M.R. Randlett, J. Appl. Phys., 39 (1968) 1525

21. H. Sugawara, T. Nagano, K. Vozumi and A. Kinbara, Thin Solid Films, 14 (1972) 349

22. K. Barua and D.C. Barua, Indian J. Pure \& Applied Phys., 14 (1976) 496

23. G. Wedler and R. Chander, Thin Solid Films, 64 (1980) 53

24. A.P. Thakoor, R. Suri, S.K. Suri and K.L. Chopra, J. Appl. Phys., 46 (1975) 4777

25. V.V.R. Narasimha Rao, S. Mohan and P. Jayarama Reddy, Thin Solid Films, 42 (1977) 283

26. V. Damorada Das and J.C. Mohanty, J. Appl. Phys., 54 (1983) 977

27. M. Bedda, S. Messaadi, C. Pichard and A.J. Tosser, J. Mater. Sci., 21 (1986) 2643

28. J.B. Thompson, Thin Solid Films, 18 (1973) 77

29. G.C. Jain and B.S. Verma, Thin Solid Films, 15 (1973) 191

30. R. Suri, A.P. Thakoor and K.L. Chopra, J. Appl. Phys., 46 (1975) 2574

31. K.L. Chopra and A.P. Thakoor, J. Appl. Phys., 49 (1978) 2857

32. F.J. Blatt and W.J. Lucke, Philos. Mag., 15 (1967) 649

33. W. Worobey, P. Lindenfeld and B. Serin, in Basic Problems in Thin Films Physics (Eds: R. Niedermayer and H. Mayer, Vandenhoeck 2 Ruprecht, Gottingen, 1966) p 601

34. R.P. Huebener, Phys. Rev. A, 136, (1964) 1740

35. J. Gouault, J. Phys., 28 (1967) 931

36. C. Reale, Phys. Lett. A, 50 (1974) 53

37. H.Y. Yu and W.F. Leonard, J. Appl. Phys., 44 (1973) 5324

38. R.L. Longbrake and S.J. Brient, Thin Solid Films, 43 (1977) 343

39. C.R. Tellier and A.J. Tosser, Thin Solid Films, 43 (1977) 261

40. C.R. Tellier, Vacuum, 28 (1978) 321

41. J.M. Chauvineau and C. Pariset, Surf. Sci., 36 (1973) 1955

42. R.E. Hummel and H.F. Geier, Thin Solid Films, 25 (1975) 335

43. C.R. Tellier, Thin Solid Films, 51 (1978) 311

44. C.R. Pichard, C.R. Tellier and A.J. Tosser, J. Phys. F, 10 (1980) 2009

45. C.R. Tellier, J. Mater. Sci., 23 (1988) 1464

46. P. Beckmann and A. Spizzichino, The Scattering of Electromagnetic Waves from Rough Surfaces, (Pergamon Press, London, 1963) chaps. 3 \& 5. 

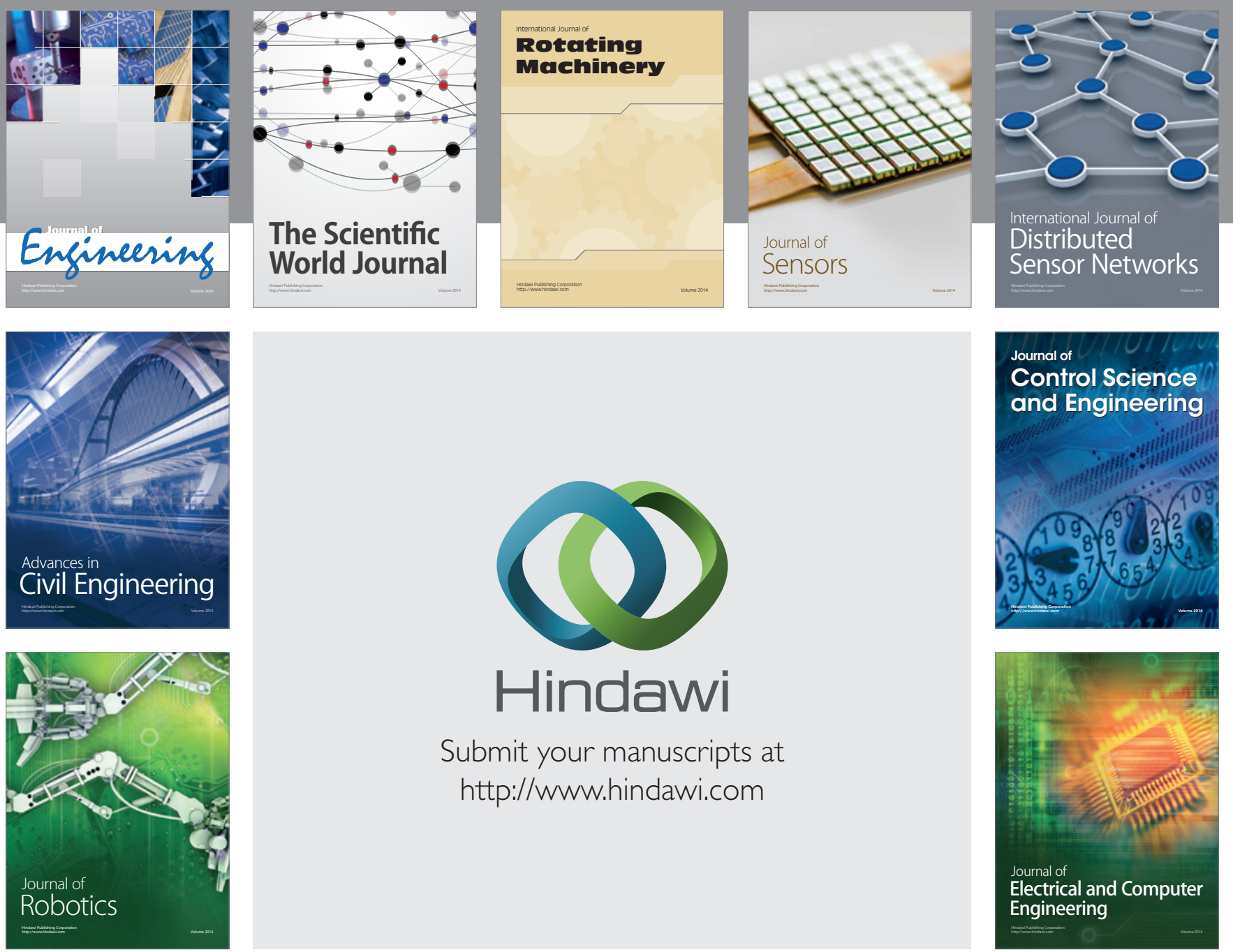

Submit your manuscripts at

http://www.hindawi.com
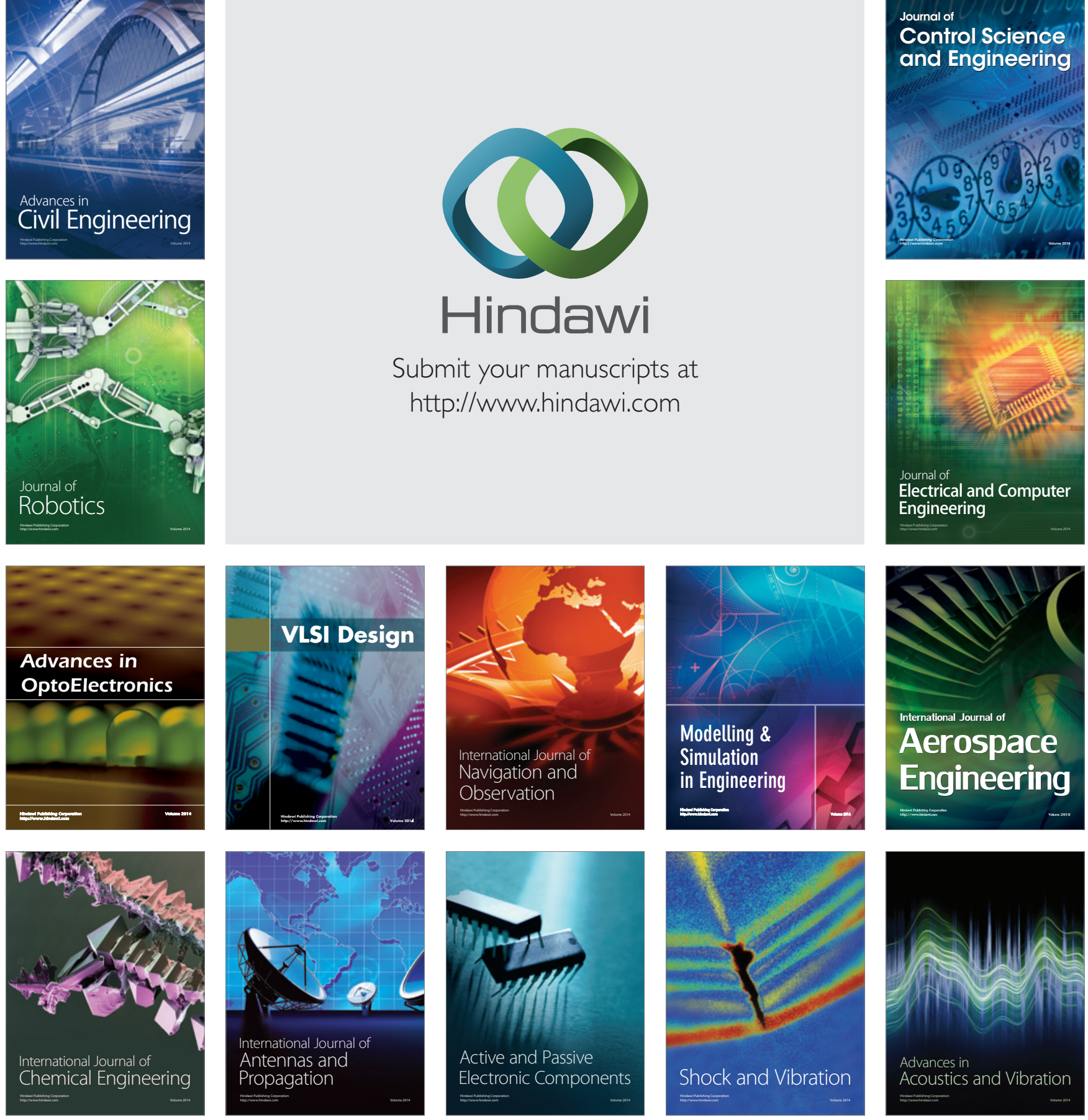